This item was submitted to Loughborough's Research Repository by the author.

Items in Figshare are protected by copyright, with all rights reserved, unless otherwise indicated.

\title{
Synthetic velocity gradient tensors and the identification of statistically significant aspects of the structure of turbulence
}

PLEASE CITE THE PUBLISHED VERSION

https://doi.org/10.1103/PhysRevFluids.2.084607

\section{PUBLISHER \\ (C) American Physical Society}

\section{VERSION}

AM (Accepted Manuscript)

\section{PUBLISHER STATEMENT}

This work is made available according to the conditions of the Creative Commons Attribution-NonCommercialNoDerivatives 4.0 International (CC BY-NC-ND 4.0) licence. Full details of this licence are available at: https://creativecommons.org/licenses/by-nc-nd/4.0/

\section{LICENCE}

CC BY-NC-ND 4.0

\section{REPOSITORY RECORD}

Keylock, Chris. 2017. "Synthetic Velocity Gradient Tensors and the Identification of Statistically Significant Aspects of the Structure of Turbulence". Loughborough University. https://hdl.handle.net/2134/33288. 


\title{
Synthetic velocity gradient tensors and the identification of significant aspects of the structure of turbulence
}

\author{
Christopher J. Keylock \\ Sheffield Fluid Mechanics Group and Department of Civil and Structural Engineering, \\ University of Sheffield, Sheffield, S1 3JD, U.K.*
}

(Dated: August 4, 2017)

\begin{abstract}
A method is presented for deriving random velocity gradient tensors given a source tensor. These synthetic tensors are constrained to lie within mathematical bounds of the non-normality of the source tensor, but we do not impose direct constraints upon scalar quantities typically derived from the velocity gradient tensor and studied in fluid mechanics. Hence, it becomes possible to ask hypotheses of data at a point regarding the statistical significance of these scalar quantities. Having presented our method and the associated mathematical concepts, we apply it to homogeneous, isotropic turbulence to test the utility of the approach for a case where the behavior of the tensor is understood well. We show that, as well as the concentration of data along the Vieillefosse tail, actual turbulence is also preferentially located in the quadrant where there is both excess enstrophy $(\mathrm{Q}>0)$ and excess enstrophy production $(\mathrm{R}<0)$. We also examine the topology implied by the strain eigenvalues and find that for the statistically significant results there is a particularly strong relative preference for the formation of disc-like structures in the $(\mathrm{Q}<0, \mathrm{R}<0)$ quadrant. With the method shown to be useful for a turbulence that is already understood well, it should be of even greater utility for studying complex flows seen in industry and the environment.
\end{abstract}

\footnotetext{
* Visiting Researcher, Department of Aeronautics, Imperial College London, London, SW7 2AZ, U.K.; c.keylock@sheffield.ac.uk
} 


\section{INTRODUCTION}

Synthetic velocity gradient tensors for incompressible turbulence, formed by imposing a divergence-free constraint on a $3 \times 3$ set of independent, Gaussian random variables, have been used for some time for discriminating between dynamic and kinematic features of turbulence. For example, as discussed further below, the development of the Vieillefosse tail [1] in $\mathrm{Q}-\mathrm{R}$ space and various eigenvector alignment properties have been shown to be dynamic quantities of the flow field through a comparison to such random, synthetic tensors $[2,3]$. However, a limitation of such analyses is that the comparison is only possible at the level of the probability distribution function for the whole flow field. There is no constructed relation between the velocity gradient tensor at a point and the properties of the synthetic data there. Hence, it is not possible with such an approach to formulate point-wise confidence limits on observed quantities.

Given the difficulty in formulating hypotheses and appropriate test statistics for examining nonlinear or chaotic aspects of observed data, a more advanced approach than Gaussian random surrogate data has been used for some time in nonlinear time series analysis. Synthetic, surrogate time series are generated that preserve the Fourier amplitudes of the original time series [4] and, additionally, the actual data values for the original time series [5]. Consequently, if $b$ surrogates are generated and one makes use of some metric of nonlinearity such as the maximal Lyapunov exponent or increment skewness [6, 7], if the value for the original data is greater or less than that for all $b$ surrogate series, then the null hypothesis may be rejected at a significance level of $2 /(b+1)$ for a two-tailed hypothesis test. Thus, in this paper, we generate 39 surrogates to permit two-tailed testing at the $5 \%$ significance level and, of course, analyse physical properties of the velocity gradient tensor, rather than measures of time series nonlinearity.

We introduced an extension to this methodology where, given such a rejection, it is possible to discern how similar to the original data the synthetic cases need to be for the hull hypothesis to be accepted by constraining the degree of phase randomization in a systematic fashion using a wavelet transform [8]. Using this approach we were able to show the significance of quantities with small absolute values in a Fokker-Planck model for

the scale-dependent evolution of the velocity increments in turbulence [9] and to propose a means for evaluating the efficacy of inlet boundary conditions in large eddy simulations 
[10]. A modified algorithm that seeks to preserve the multifractal structure of time series has recently been used to examine singuarity structure and velocity-intermittency coupling in turbulence [11].

If we can generate random tensors conditioned in some sense on the observed pointwise structure of the original data then local testing of significance becomes possible. Thus, "unusual" tensors where a significant difference in some property of the tensor exists may be identified and studied further. However, it is not necessarily obvious what these preserved or "pseudo-preserved" properties should be, or from which distribution function they should be sampled. Furthermore, any such method should attempt to minimize the arbitrariness of the selection of distribution functions for the quantities to be randomized and should generate random realizations that retain the appropriate structure of the original tensor. It is the development of an approach to tensor randomization that this paper sets out to address. Following some additional detail regarding our approach to synthetic tensor generation, we review some properties of the velocity gradient tensor, a more detailed treatment of which can be found in the review paper by Meneveau [12]. We then present some properties of the Schur transform of a matrix or tensor, which is the tool from matrix algebra used in this study to underpin the randomization algorithm. Having developed our approach, we proceed to example analyses for homogeneous, isotropic turbulence (HIT) because it is a case that is already understood well. We show that in addition to the Vieillefosse tail, real turbulence has a preferential occupation of the second quadrant $\mathrm{Q}>0, \mathrm{R}<0$ of the $\mathrm{Q}-\mathrm{R}$ diagram. We then look at locations where the structure of the intermediate eigenvalue of the strain rate tensor is significantly different to the surrogates and contrast the underlying turbulence structure for these cases relative to the global distribution functions for HIT. Given that HIT provides the basis for much of turbulence theory, our approach will be of even greater potential utility when studying flows for which there is less knowledge of the dynamics and where placing statistical confidence on observed baheviors will, as a consequence, be of greater utility. This includes inhomogeneous and anisotropic flows seen in the environment and industry. 


\section{A. Synthetic data for hypothesis testing}

With an increasing ability to resolve and study the velocity gradient tensor in experiments $[13,14]$ to accompany the long history of such numerical work [15], as well as the use of flow structure visualization criteria based on the velocity gradient tensor in applied studies [16-18], inferring the statistical significance of observed results compared to suitable null models is important for placing confidence on, and thereby interpreting, observed results. In particular, as noted above, previous studies have identified differences between the properties of real velocity gradient tensors and those realized from Gaussian random tensors [3], but this is only possible at the scale of the distribution function because there is no intrinsic relation between an individually resolved tensor and a set of orthonormalized, Gaussian random numbers. The aim of this paper is to develop a method where each realized random tensor is suitably constrained to properties of the observed tensor. This philosophy may be contrasted with that of the older approach by stating the null hypotheses in each case, commencing with the Gaussian case:

- $H_{0}$ : There is no significant difference between a moment (mean, standard deviation) of a property of the observed velocity gradient tensors and the same property measured on orthonormalized, Gaussian, random tensors;

- $H_{0}$ : There is no significant difference between a property of the observed velocity gradient tensor and the same property measured on random tensors constrained to preserve basic properties of the observed tensor.

In these two null hypotheses, a 'property' is taken to be any quantity obtained locally based on the velocity gradient tensor, such as the alignment of the vorticity vector and the straining eigenvectors, the rate of strain production, etc.

The latter null hypothesis is more relevant physically and is the only one of the two that can be used if the interest is in measurements at a particular point in space and time as the random tensors are sufficiently "matched" to the observed case that it is meaningful to make a local comparison. As a consequence, such random tensors are expected to be of particular utility in non-homogeneous and anisotropic flows where spatial and temporal averaging is less useful than is the case in homogeneous, isotropic turbulence.

Our intention is to formulate synthetic tensors that approximately preserve, statistically, 
the marginal distributions of basic properties of the tensors, but not the joint distribution of these quantities, or more refined properties. As a consequence, our synthetic tensors are useful for studying these very same aspects of the tensor. In light of these considerations, there are three guiding principles behind our approach:

1. The synthetic tensor should replicate the observed level of compressibility (to extend our approach beyond merely examining incompressible turbulence, and to deal with the experimental difficulty of observing exact incompressibility [19]);

2. Because of their long-standing importance in the analysis of the velocity gradient tensor [1, 20-23], it is desirable that we constrain the synthetic tensors so as to approximate the invariants of the characteristic equation of the velocity gradient tensor. These quantities known as $\mathrm{P}, \mathrm{Q}$, and $\mathrm{R}$ are described in the next section, but note that principle (1) amounts to exactly replicating the observed $\mathrm{P}$, while we will approximate $\mathrm{Q}$ and $\mathrm{R}$ so that properties of the joint distribution of the two may be studied;

3. Strain and rotation (vorticity) are key concepts in turbulence and in the analysis of the tensor. In particular, as explained in Section II, Q can be viewed as the excess of enstrophy over total strain and $\mathrm{R}$ as the excess of strain production over enstrophy production. Given the physical interest in these quantities, the synthetic tensor algorithm should not directly constrain such terms. Thus, they may be analyzed rather than matched in the synthetic data. To achieve this, we decompose the tensor in an alternate way to the Hermitian/skew-Hermitian decomposition into strain and rotation that fluid mechanicists usually adopt.

Given these principles, the rest of the manuscript is largely concerned with the development of such a method, a demonstration that it approximates the values for $\mathrm{Q}$ and $\mathrm{R}$ in homogeneous, isotropic turbulence and then two example analyses are undertaken. The first is based on the joint distribution function for $\mathrm{Q}$ and $\mathrm{R}$, and the second studies the eigenvalues of the strain tensor. Before presenting our method, we first review some basic properties of the velocity gradient tensor. 


\section{THE VELOCITY GRADIENT TENSOR IN TURBULENCE}

The velocity gradient tensor, $\mathbf{A}$ is given by

$$
\mathbf{A}=\left(\begin{array}{lll}
\partial u_{1} / \partial x_{1} & \partial u_{1} / \partial x_{2} & \partial u_{1} / \partial x_{3} \\
\partial u_{2} / \partial x_{1} & \partial u_{2} / \partial x_{2} & \partial u_{2} / \partial x_{3} \\
\partial u_{3} / \partial x_{1} & \partial u_{3} / \partial x_{2} & \partial u_{3} / \partial x_{3}
\end{array}\right),
$$

where $x$ is a distance along an orthogonal axis, $u$ is a velocity component, and the subscripts indicate the relevant, orthogonal orientation. From this, one may define strain, rotation and vorticity terms, given respectively by

$$
\begin{aligned}
\mathbf{S} & =\frac{1}{2}\left(\mathbf{A}+\mathbf{A}^{*}\right) \\
\mathbf{\Omega} & =\frac{1}{2}\left(\mathbf{A}-\mathbf{A}^{*}\right) \\
\omega_{i} & =\epsilon_{i j k} \Omega_{j k},
\end{aligned}
$$

where $\epsilon_{i j k}$ is the Levi-Civita symbol and the $*$ superscript is the conjugate transpose. The role of $\mathbf{A}$ in the dynamics of turbulence may be made explicit by taking the spatial gradient of the Navier-Stokes equations:

$$
\frac{\partial}{\partial t} \mathbf{A}+\mathbf{u} \cdot \nabla \mathbf{A}=-\mathbf{A}^{2}-\mathbf{E}+\nu \nabla^{2} \mathbf{A}
$$

where $\mathbf{u}$ is the velocity, $\mathbf{E}$ is the Hessian of the kinematic pressure field, i.e. $E_{i j}=\frac{\partial^{2} p}{\partial x_{i} \partial x_{j}}$, where $p$ is the pressure.

It is common to investigate the properties of $\mathbf{A}$ with respect to its characteristic polynomial

$$
\lambda_{i}^{3}+\mathrm{P} \lambda_{i}^{2}+\mathrm{Q} \lambda_{i}+\mathrm{R}=0
$$

where for an incompressible flow, because $\operatorname{tr}(\mathbf{A})=0$, and $\mathrm{P}=\sum_{i} \lambda_{i}$, it follows that $\mathrm{P}=0$. The second and third invariants, $\mathrm{Q}$ and $\mathrm{R}$, and their associated evolution equations are studied frequently as they provide information on the relative importance of strain and enstrophy, as well as their production in the flow [21, 24]:

$$
\begin{aligned}
& \mathrm{Q}=-\frac{1}{2} \operatorname{tr}\left(\mathbf{A}^{2}\right) \equiv \frac{1}{2}\left(-\operatorname{tr}\left(\mathbf{\Omega}^{2}\right)-\operatorname{tr}\left(\mathbf{S}^{2}\right)\right) \equiv \frac{1}{2}\left(\|\mathbf{\Omega}\|^{2}-\|\mathbf{S}\|^{2}\right) \\
& \mathrm{R}=-\operatorname{Det}(\mathbf{A}) \equiv-\operatorname{Det}(\mathbf{S})-\operatorname{tr}\left(\mathbf{\Omega}^{2} \mathbf{S}\right)
\end{aligned}
$$

where $\|\ldots\|$ is the Frobenius norm, i.e. $\|\mathbf{S}\|=\operatorname{tr}\left(\mathbf{S} \times \mathbf{S}^{*}\right)^{\frac{1}{2}}$. The associated $\mathrm{Q}-\mathrm{R}$ diagram has played an important role in our understanding of the nature of HIT and other types of turbulent flow $[1,23,25]$, and correctly resolving the $\mathrm{Q}-\mathrm{R}$ diagram has been used recently 
as a means of checking the effective resolution of numerical simulations [26]. In addition, positive values of $\mathrm{Q}$ are used in more applied studies as a local approach to identify vortical structures in the flow field [16, 17, 27, 28]. Consequently, a suite of analyses for turbulence may be undertaken given a database of velocity gradient tensor fields, obtained numerically [29] or experimentally [30].

\section{THE SCHUR TRANSFORM AND THE EIGENVALUE STRUCTURE OF THE VELOCITY GRADIENT TENSOR}

In this study we avoid basing a randomization on a Hermitian/skew-Hermitian decomposition of $\mathbf{A}$ into strain and rotation components and instead adopt the Schur transform [31]. In this way, we do not undertake direct manipulation or impose direct constraints on quantities that are likely to be of prominent physical interest.

The Schur transform is given by

$$
\mathbf{A}=\mathbf{U T U}^{*},
$$

where $\mathbf{U}$ is unitary. In the case of complex eigenvalues, there are two variants of (8): the complex Schur transform where $\mathbf{U} \in \mathbb{C}$ and $\mathbf{T} \in \mathbb{C}$ is upper triangular; and, the real Schur transform where if the eigenvalues are complex, $\mathbf{U}_{r} \in \mathbb{R}$ and $\mathbf{T}_{r} \in \mathbb{R}$ is merely quasi-upper triangular. That is, in the case of a normal velocity gradient tensor with complex eigenvalues, we have

$$
\mathbf{T}_{r}=\left(\begin{array}{ccc}
\mathbb{R}\left(\lambda_{c}\right) & -\Im\left(\lambda_{c}\right) & 0 \\
\Im\left(\lambda_{c}\right) & \mathbb{R}\left(\lambda_{c}\right) & 0 \\
0 & 0 & \lambda_{r}
\end{array}\right)
$$

where $\lambda_{c}$ is the conjugate pair eigenvalue with positive imaginary part and $\lambda_{r}$ is the real eigenvalue. Of course, if all $\lambda_{i} \in \mathbb{R}$ then $\mathbf{T}_{r}$ is upper triangular and identical to the complex Schur transform. Because the real decomposition introduces this variation in the nature of the Schur matrix, T, we use the complex variant of the Schur transform in what follows so that all Schur matrices are always in an upper triangular form.

Given that we have established that $\mathbf{T}$ is upper triangular, we may write that

$$
\begin{aligned}
\mathbf{T} & =\left(\begin{array}{ccc}
\lambda_{1} & N_{1,2} & N_{1,3} \\
0 & \lambda_{2} & N_{2,3} \\
0 & 0 & \lambda_{3}
\end{array}\right) \\
& =\left(\begin{array}{ccc}
L_{1,1} & N_{1,2} & N_{1,3} \\
0 & L_{2,2} & N_{2,3} \\
0 & 0 & L_{3,3}
\end{array}\right) \\
& =\mathbf{L}+\mathbf{N},
\end{aligned}
$$


where $\mathbf{L}$ is a diagonal matrix of eigenvalues and $\mathbf{N}$ contains the tensor non-normality.

Thus, the Schur transform may be contrasted with an eigenvalue-eigenvector decomposition: The two are equivalent when a tensor is normal, but while the latter accommodates non-normality in the departure of the eigenvector matrix from an unitary form, $\mathbf{U}$ is always unitary and the non-normality appears explicitly in $\mathbf{N}$. Noting that a velocity gradient tensor reconstructed from $\mathbf{L}$, i.e $\mathbf{U L U}^{*}$, will be symmetric, in the case of the turbulent velocity gradient tensor, the physical interpretation of the non-normality is that it contains the asymmetric velocity gradients, resulting from non-local effects, which induce torques on the fluid element.

A major advantage of the Schur transform as the basis for randomization is therefore that the unitary form for $\mathbf{U}$ is a strong constraint, permitting classical theory to be utilized: the orthonormalization of nine zero mean, unit variance, Gaussian random numbers will work well. With a tilde denoting a synthetic variant of a particular matrix of tensor, it is clear that decisions with respect to the form of $\widetilde{\mathbf{T}}$ are more complex. A logical starting point would be to preserve the Frobenius norms of $\mathbf{N}$ and $\mathbf{L}$ as well as the value for $\mathrm{P}$ to give the required level of compressibility. However, if the eigenvalues are complex then it is not clear how the magnitudes of the real and imaginary parts of $\widetilde{\mathbf{T}}$ should be partitioned, beyond the existence of a conjugate pair in $\widetilde{\mathbf{L}}$ and a known value for $\mathrm{P}$. This means that there is relatively little to guide one's choice of sampling distribution function for defining values in $\widetilde{\mathbf{T}}$. Of course, another necessary constraint for physically relevant values for $\widetilde{\mathbf{A}}$ is that $\widetilde{\mathbf{U T}} \widetilde{\mathbf{U}^{*}} \in \mathbb{R}$, which adds additional complexity to the formulation of $\widetilde{\mathbf{U}}$.

Because of these potential difficulties, we formulate a less direct approach where constraints follow from the form for bounds on the behavior of the tensor. Hence, we make use of mathematical properties of the tensor to derive appropriately constrained realizations. More specifically, we bound the observed non-normality (given by $\mathbf{N}$ ) and replicate the expected level of compressibility. 


\section{SYNTHETIC TENSOR ALGORITHM}

\section{A. Non-normality of a matrix or tensor and relevant bounds}

By adopting the Schur decomposition, we can obtain relevant mathematical bounds on the degree of permitted randomization. This is because commonly adopted definitions of non-normality may be expressed in terms of $\|\mathbf{N}\|$ as may the bounds on the maximum permissible non-normality. Hence, we can use these to perturb the tensor to an appropriate degree. Given a velocity-gradient tensor, A, there are two common ways to define its nonnormality. The first is based on the definition of a normal matrix as one where $\mathbf{A}^{*} \mathbf{A}=\mathbf{A} \mathbf{A}^{*}$, leading to a measure for the departure from normality, due to Henrici [32], as one based on the Frobenius norm of the difference:

$$
\eta_{H} \equiv\|\mathbf{H}\|=\left\|\mathbf{A}^{*} \mathbf{A}-\mathbf{A} \mathbf{A}^{*}\right\|
$$

An alternative approach is to define the complex Schur decomposition as used above, $\mathbf{A}=$ UTU*. It then follows that $\mathbf{A}$ is normal if $\mathbf{U L U}^{*}=\mathbf{U T U}^{*}$ and, therefore, the Schur non-normality is

$$
\eta_{C}=\|\mathbf{N}\|
$$

A number of studies have formulated bounds for the relation between $\eta_{H}$ and $\eta_{C}$ and two such bounds that constrain the behavior of $\mathbf{A}$ given $\eta_{C}$ are $[32,33]$ :

$$
\begin{aligned}
\eta_{H}^{(l)} & \leq \eta_{H} \leq \eta_{H}^{(u)} \\
\eta_{H}^{(l)} & =\left(\frac{\eta_{C}}{2^{\frac{1}{4}}}\right)^{2} \\
\eta_{H}^{(u)} & =\sqrt{6} \eta_{C}\|\mathbf{A}\|
\end{aligned}
$$

As described above, the typical additive decomposition of the velocity gradient tensor is based on strain, $\mathbf{S}$, and rotation, $\boldsymbol{\Omega}$. Substitution of $\mathbf{A}=\mathbf{S}+\boldsymbol{\Omega}$ into (11) and grouping of terms gives

$$
\begin{aligned}
\mathbf{H} & =\left(\mathbf{S}^{*} \mathbf{S}-\mathbf{S S}^{*}\right)+\left(\mathbf{S}^{*} \boldsymbol{\Omega}-\mathbf{S} \boldsymbol{\Omega}^{*}\right) \\
& +\left(\boldsymbol{\Omega}^{*} \mathbf{S}-\boldsymbol{\Omega} \mathbf{S}^{*}\right)+\left(\boldsymbol{\Omega}^{*} \boldsymbol{\Omega}-\boldsymbol{\Omega} \boldsymbol{\Omega}^{*}\right)
\end{aligned}
$$

where the symmetric and skew-symmetric nature of $\mathbf{S}$ and $\boldsymbol{\Omega}$ means that the first and last terms have all zero elements. Furthermore, the second and third terms are equal, meaning 
that, arbitrarily choosing to work with the third, we may write that $\mathbf{H}=2\left(\boldsymbol{\Omega}^{*} \mathbf{S}-\mathbf{S} \boldsymbol{\Omega}^{*}\right)$ and, therefore,

$$
\eta_{H}=2\left\|\mathbf{\Omega}^{*} \mathbf{S}-\mathbf{S} \mathbf{\Omega}^{*}\right\|
$$

Hence, we establish in (15) that manipulation of $\left(\boldsymbol{\Omega}^{*} \mathbf{S}\right)$ can be directly related to bounds on the relations between $\eta_{H}$ and $\eta_{C}$ in (13) using (11) and substituting in expressions for the rotation and strain tensors.

\section{B. Some properties of the $\Omega^{*} S$ tensor}

Note that irrespective of whether or not $\mathrm{P}=0$, the eigenvalues of $\left(\boldsymbol{\Omega}^{*} \mathbf{S}\right)$ sum to zero as a direct consequence of the traceless nature of $\boldsymbol{\Omega}$. Recalling equations (6) and (7) and introducing an $(\Omega S)$ subscript to identify that we are talking about the invariants of the characteristic equation for $\Omega^{*} \mathbf{S}$, then this means that $\mathrm{P}_{(\Omega S)}=0$. Furthermore, because $\Omega$ is singular (it has a zero eigenvalue), so is $\left(\Omega^{*} \mathbf{S}\right)$, meaning that $\operatorname{det}\left(\boldsymbol{\Omega}^{*} \mathbf{S}\right)=0$. Therefore, $\mathrm{R}_{(\Omega S)}=0$ and the characteristic equation may be expressed just with its linear term, $\mathrm{Q}_{(\Omega S)}$. There are two situations that pertain, which are made explicit by writing $\mathrm{Q}_{(\Omega S)}$ in terms of its own Hermitian/skew-Hermitian decomposition:

$$
\begin{aligned}
\mathrm{Q}_{(\Omega S)} & =\|\Psi\|^{2}-\|\Theta\|^{2} \\
\Theta & =\frac{1}{2}\left[\left(\boldsymbol{\Omega}^{*} \mathbf{S}\right)+\left(\boldsymbol{\Omega}^{*} \mathbf{S}\right)^{*}\right] \\
\Psi & =\frac{1}{2}\left[\left(\boldsymbol{\Omega}^{*} \mathbf{S}\right)-\left(\boldsymbol{\Omega}^{*} \mathbf{S}\right)^{*}\right],
\end{aligned}
$$

and are given as

- $\mathrm{Q}_{(\Omega S)}>0$, which means that the eigenvalues for $\left(\Omega^{*} \mathbf{S}\right)$ are complex and of the form $\mathbf{L}_{(\Omega S)}=\left(\begin{array}{lll}0+\lambda^{(\Omega S)} \text { i } 0 & 0 \\ 0 & 0-\lambda^{(\Omega S)} & 0 \\ 0 & 0 & 0\end{array}\right)$

- $\mathrm{Q}_{(\Omega S)}<0$, which means that the eigenvalues for $\left(\boldsymbol{\Omega}^{*} \mathbf{S}\right)$ are real and of the form $\mathbf{L}_{(\Omega S)}=\left(\begin{array}{lll}\lambda^{(\Omega S)} & 0 & 0 \\ 0 & -\lambda^{(\Omega S)} & 0 \\ 0 & 0 & 0\end{array}\right)$.

\section{Randomization of $\left(\boldsymbol{\Omega}^{*} \mathbf{S}\right)$}

We write the Schur transform of $\left(\boldsymbol{\Omega}^{*} \mathbf{S}\right)$ as

$$
\left(\Omega^{*} \mathbf{S}\right)=\mathbf{U}_{(\Omega S)} \mathbf{T}_{(\Omega S)} \mathbf{U}_{(\Omega S)}^{*},
$$


The Schur transform is not unique as the ordering of the eigenvalues on the diagonal is not prescribed. However, we may undertake rotations such that the zero eigenvalue is located at $L_{(\Omega S)}(3,3)$, given that $\mathbf{T}_{(\Omega S)}=\mathbf{L}_{(\Omega S)}+\mathbf{N}_{(\Omega S)}$, by imposing a constraint that the block of values to be rotated are those outside the unit disk [34]. That is, we seek the positions, $m$, where there are non-zero eigenvalues and if $\left|\lambda_{m}^{(\Omega S)}\right|<1$ pre-multiply $\mathbf{T}_{(\Omega S)}$ by a constant, $k$ such that $\left|k \lambda_{m}\right|>1$. We then undertake the appropriate Schur rotation and divide through by $k$ to return to the original eigenvalues. Having now located the zero eigenvalue at $L_{(\Omega S)}(3,3)$ we can simplify subsequent notation as the salient locations for the non-zero eigenvalues are set to be $L_{(\Omega S)}(1,1)$ and $L_{(\Omega S)}(2,2)$.

\section{Randomization of the unitary matrix, $\widetilde{\mathbf{U}}_{(\Omega S)}$}

The unitary constraint is a strong one for randomization, making treatment of this part of the Schur transform relatively straightforward. We form a random unitary matrix, $\widetilde{\mathbf{U}}_{(\Omega S)}$, as indicated by the tilde, by the orthonormalization of a $3 \times 3$ Gaussian random matrix, $\mathbf{G}$, where each element, $G_{i, j}$, is sampled independently from a Gaussian variate with zero mean and a standard deviation of one before the orthonormalization step.

\section{Randomization of the non-normal matrix, $\widetilde{\mathbf{N}}_{(\Omega S)}$}

The manner in which the difficult task of formulating a direct randomization of eigenvalues is circumvented in our approach then becomes clear: Because we are not undertaking the randomization directly on $\mathbf{A}$, but on $\left(\boldsymbol{\Omega}^{*} \mathbf{S}\right)$, we can fix the eigenvalues of $\left(\boldsymbol{\Omega}^{*} \mathbf{S}\right)$, i.e.

$\mathbf{L}_{(\Omega S)}$, but randomize our non-normality term, $\widetilde{\mathbf{N}}_{(\Omega S)}$ and, as already described, our unitary matrix, $\widetilde{\mathbf{U}}_{(\Omega S)}$, to give

$$
\left(\widetilde{\mathbf{\Omega}^{*} \mathbf{S}}\right)=\widetilde{\mathbf{U}}_{(\Omega S)}\left(\mathbf{L}_{(\Omega S)}+\left[\widetilde{\mathbf{N}}_{(\Omega S)}\left|\| \mathbf{N}_{(\Omega S)}\right| \mid\right]\right) \widetilde{\mathbf{U}}_{(\Omega S)}^{*}
$$

Here we have written $\left[\widetilde{\mathbf{N}}_{(\Omega S)} \mid\left\|\mathbf{N}_{(\Omega S)}\right\|\right]$ in full to highlight that the randomization of the non-normal part of the Schur decomposition is conditioned on the preservation of the original Frobenius norm for this part. On this understanding, we simplify notation in what follows so that $\widetilde{\mathbf{N}}_{(\Omega S)} \equiv\left[\widetilde{\mathbf{N}}_{(\Omega S)} \mid\left\|\mathbf{N}_{(\Omega S)}\right\|\right]$.

Two approaches to the randomization of $\mathbf{N}_{(\Omega S)}$ are suggestive: 
- Randomize all three values in $\mathbf{N}_{(\Omega S)}$ independently, subject to the Frobenius norm constraint, to give $\widetilde{\mathbf{N}}_{(\Omega S)}^{(3)}$;

- Randomize independently, subject to the Frobenius norm constraint, just the two values in the third column of $\mathbf{N}_{(\Omega S)}$ (i.e. those associated with the zero eigenvalue at $L_{3,3}$ in the subsequent matrix multiplication), to give $\widetilde{\mathbf{N}}_{(\Omega S)}^{(2)}$.

In the former case, we proceed by generating two uniformly distributed, real, random variates:

- The first is $0 \leq \widetilde{N}_{1,2}^{(3)} \leq\left\|\mathbf{N}_{(\Omega S)}\right\|$;

- The second is $0 \leq \widetilde{N}_{1,3}^{(3)} \leq \sqrt{\left(\left\|\mathbf{N}_{(\Omega S)}\right\|^{2}-\left[\widetilde{N}_{1,2}^{(3)}\right]^{2}\right)}$; and,

- It then follows that the third value is given by

$$
\widetilde{N}_{2,3}^{(3)}=\sqrt{\left(\left\|\mathbf{N}_{(\Omega S)} \mid\right\|^{2}-\left(\left[\widetilde{N}_{1,2}^{(3)}\right]^{2}+\left[\widetilde{N}_{1,3}^{(3)}\right]^{2}\right)\right)} .
$$

For the latter case, $N_{1,2}$ is not randomized, meaning that:

- We generate $0 \leq \widetilde{N}_{1,3}^{(2)} \leq \sqrt{\left(\left\|\mathbf{N}_{(\Omega S)}\right\|^{2}-N_{1,2}^{2}\right)}$; and,

- This leaves $\widetilde{N}_{2,3}^{(2)}=\sqrt{\left(\left\|\mathbf{N}_{(\Omega S)} \mid\right\|^{2}-\left(\left[N_{1,2}^{(2)}\right]^{2}+\left[\widetilde{N}_{1,3}^{(2)}\right]^{2}\right)\right)}$.

Finally, we allocate signs to all the $\widetilde{N}_{i, j}$ by simply converting randomly generated values that are less than or greater than the median of a selected distribution function into negative and positives, respectively. This step is required because the Frobenius norm constraint provides no information on these signs.

Consequently, using these rules, combined with (17) and (18), we obtain our random quantity, $\widetilde{\Omega^{*} \mathbf{S}}$. As we do not necessarily wish to assume an incompressible flow where the first invariant of the characteristic equation of $\mathbf{A}, \mathrm{P}=0$, we have that $\mathrm{P}=\sum_{i} \lambda_{i}$ as an additional constraint. Hence, we seek a strain tensor with elements $a$ to $f$ and a rotation tensor with elements $g, h$ and $m$ such that

$$
\begin{aligned}
\left(\begin{array}{ccc}
a & d & e \\
d & b & f \\
e & f & c
\end{array}\right)\left(\begin{array}{ccc}
0 & g & h \\
-g & 0 & m \\
-h & -m & 0
\end{array}\right) & =\widetilde{\mathbf{\Omega}^{*} \mathbf{S}} \\
a+b+c & =\mathrm{P},
\end{aligned}
$$

Clearly, given such a solution to (19), we have

$$
\widetilde{\mathbf{A}}=\left(\begin{array}{ccc}
a & d+g & e+h \\
d-g & b & f+m \\
e-h & f-m & c
\end{array}\right) .
$$




\section{Rejection sampling for the non-normality constraint}

If we define $\tilde{\eta}_{C}$ and $\tilde{\eta}_{H}$ as the Schur-based non-normality, and the Henrici non-normality for $\widetilde{\mathbf{A}}$, then the final step of our algorithm is to impose the bounds given in (13). Thus, the realized tensor, $\tilde{\mathbf{A}}$, is a possible variant of $\mathbf{A}$ given knowledge of the bounds on its non-normality.

We impose (13) by rejection sampling. That is, rather than making the constraints in (19) more complex, we reject $\tilde{\mathbf{A}}$ if the bounds are not fulfilled. Because of the relation established between these bounds and $\left(\boldsymbol{\Omega}^{*} \mathbf{S}\right)$ given in (15), typically, a legitimate choice for $\tilde{\mathbf{A}}$ is obtained after one or two attempts as is shown in the next section. That is, the value of $\tilde{\eta}_{H}$ for the synthetic tensor $\tilde{\mathbf{A}}$ lies within the bounds for the actual non-normality, $\eta_{H}$, of A.

\section{TESTING THE GENERATION SCHEME WITH AN EXAMPLE CASE}

Our choice for a test strain tensor could be completely arbitrary, but to provide some realism, we choose one where the eigenvalues sum to zero, and where they are in a ratio $3: 1:-4$, as may be observed in homogeneous, isotropic turbulence [35]. An example is

$$
\mathbf{S}_{\mathbf{A}_{1}}=\left(\begin{array}{ccc}
-0.375 & -0.750 & -0.188 \\
-0.750 & -0.125 & -0.563 \\
-0.188 & -0.563 & 0.500
\end{array}\right)
$$

which, combined with

$$
\boldsymbol{\Omega}_{\mathbf{A}_{1}}=\left(\begin{array}{ccc}
0.000 & -0.125 & 0.438 \\
0.125 & 0 & 0.313 \\
-0.438 & -0.313 & 0.000
\end{array}\right)
$$

gives

$$
\mathbf{A}_{1}=\left(\begin{array}{ccc}
-0.375 & -0.875 & 0.250 \\
-0.625 & -0.125 & -0.250 \\
-0.625 & -0.875 & 0.500
\end{array}\right)
$$

with $\mathrm{Q}_{\left(\mathbf{A}_{1}\right)}=-\frac{13}{16}$ and $\mathrm{R}_{\left(\mathbf{A}_{1}\right)}=\frac{9}{48}$. The opposite quadrant in $\mathrm{Q}-\mathrm{R}$ space can be realised readily in this case by simply doubling $\boldsymbol{\Omega}_{\mathbf{A}_{1}}$ :

$$
\mathbf{A}_{2}=\left(\begin{array}{lll}
-0.375 & -1.000 & 0.688 \\
-0.500 & -0.125 & 0.063 \\
-1.064 & -1.189 & 0.500
\end{array}\right)
$$

with $\mathrm{Q}_{\left(\mathbf{A}_{2}\right)}=0.104$ and $\mathrm{R}_{\left(\mathbf{A}_{2}\right)}=-0.130$.

The consequences of choosing to impose, or not to impose, rejection sampling on the algorithm are shown in Fig. 1, where between $20 \%$ and $30 \%$ of the generated surrogates exceed the Eberlein bound without adopting rejection sampling. It is also clear that there is 


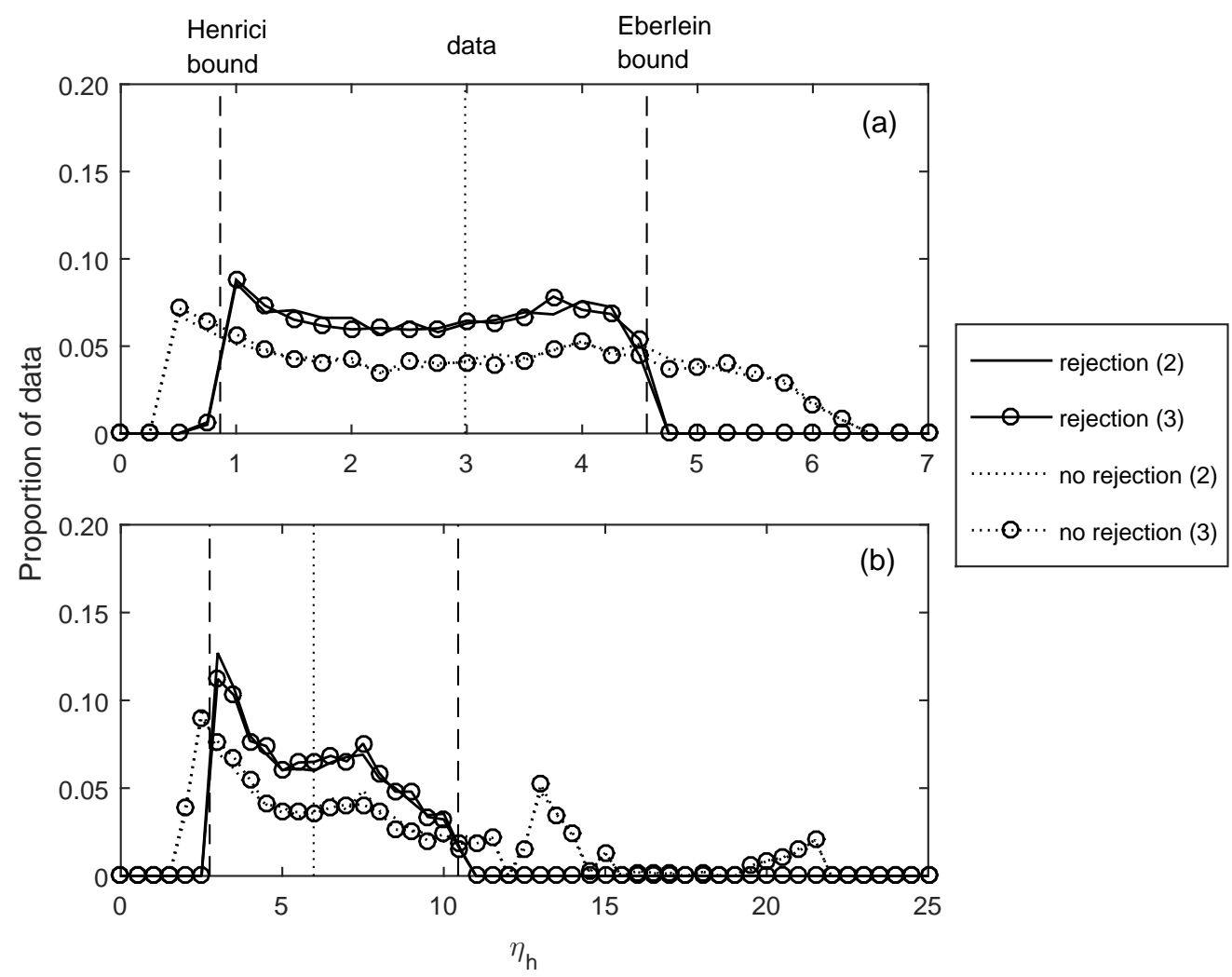

FIG. 1. Histograms of the distribution of $\tilde{\eta}_{H}$ values for realizations of example tensors $\mathbf{A}_{1}$ in panel (a) and of $\mathbf{A}_{2}$ in panel (b). In each panel, the black lines are based on rejection sampling to impose the bounds on $\tilde{\eta}_{H}$, while the dotted line indicates the consequences of not imposing (13). The lines without circles are those where we randomize two values in $\tilde{\mathbf{N}}_{(\Omega S)}$, while those with circles are based on randomization of all three values. There is very little difference between the results for $\tilde{\mathbf{N}}_{\Omega S}^{(2)}$ and $\tilde{\mathbf{N}}_{\Omega S}^{(3)}$. The vertical dashed lines show the values for $\eta_{H}^{(l)}$ and $\eta_{H}^{(u)}$ and the vertical dotted line is $\eta_{H}$ for the original tensor $\left(\mathbf{A}_{1}\right.$ or $\left.\mathbf{A}_{2}\right)$.

very little difference in the results from the use of the $\widetilde{\mathbf{N}}_{(\Omega S)}^{(2)}$ or the $\widetilde{\mathbf{N}}_{(\Omega S)}^{(3)}$ scheme. If rejection sampling is adopted, Fig. 2 shows that $\sim 97 \%$ of successful realizations are generated in five or fewer attempts. 

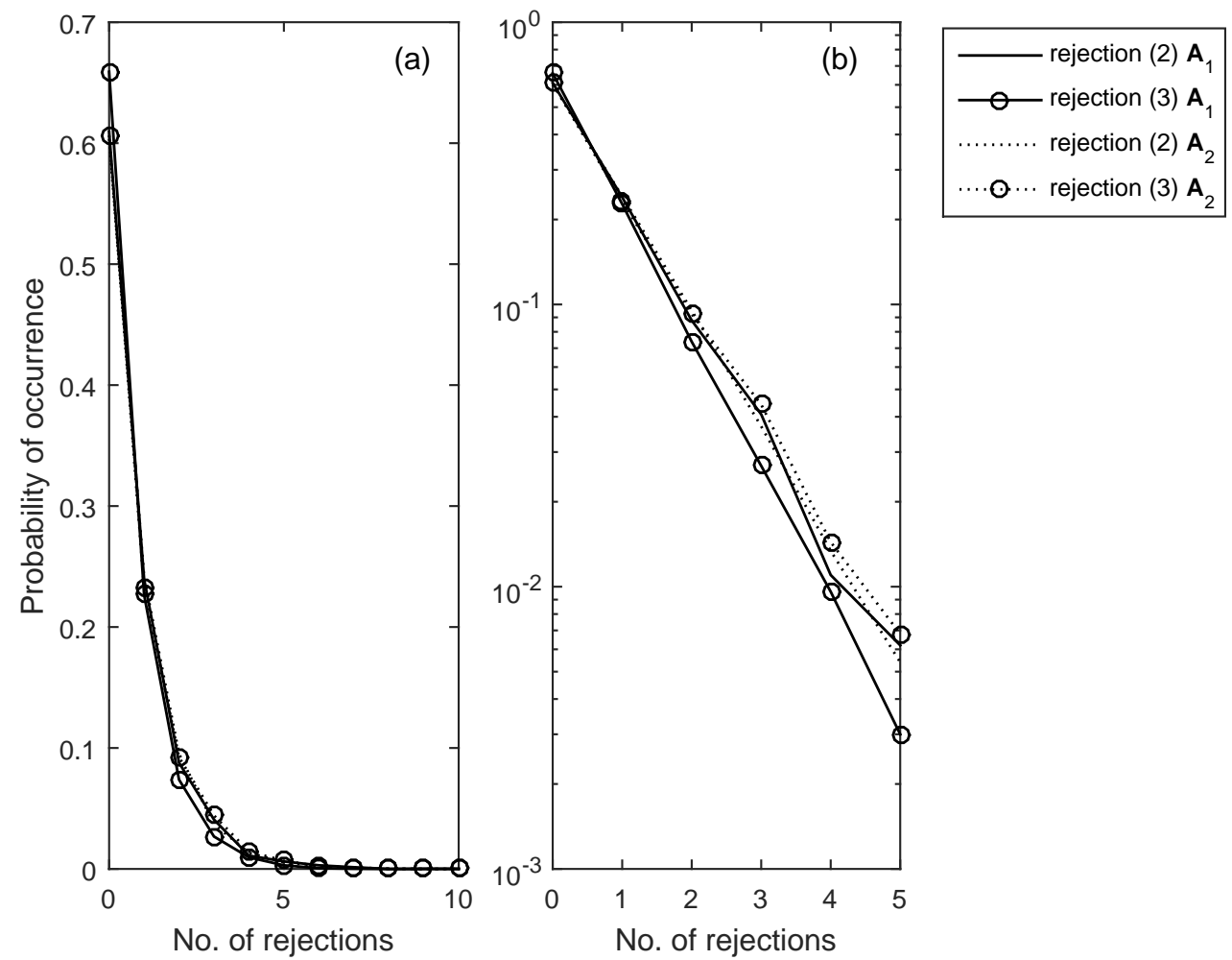

FIG. 2. Histograms showing the exponential distribution of the number of rejected samples before an acceptable sample is generated. Panel (b) shows the same data as in the first panel, but on a logarithmic ordinate. The solid lines are the results for $\mathbf{A}_{1}$, while the dotted lines are for $\mathbf{A}_{2}$.

\section{APPLICATION TO HOMOGENEOUS, ISOTROPIC TURBULENCE (HIT)}

While we believe that the utility of this method will be greatest in complex flows where there is little recourse to theory to inform the behavior of the velocity gradient tensor, and hypothesis testing comes into its own, application to HIT provides a means to explore the properties of our algorithm against a well understood benchmark [12]. In particular, wellknown features of HIT VGTs such as the Vieillefosse tail [1] are not imposed structurally in our algorithm, meaning that we should expect to see significant differences in this region (this is a true property of HIT that differs from randomized data).

This study makes use of velocity gradient tensors extracted from the Johns Hopkins numerical simulation of HIT at a Taylor Reynolds number of 433 [29], which has become 

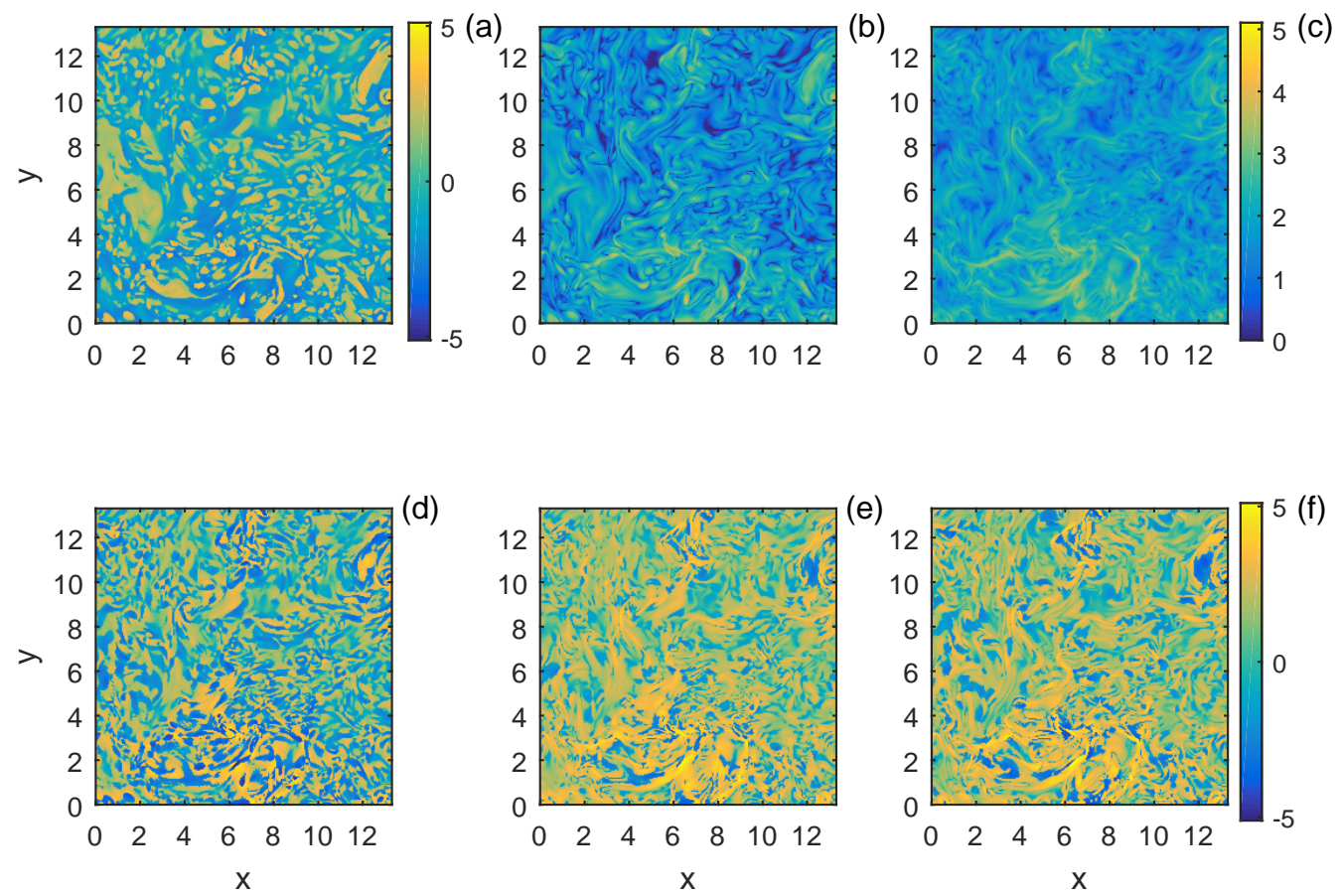

FIG. 3. Fields of six scalar quantities derived from the velocity gradient tensor. The top row shows Q (a) and its constituents: $\boldsymbol{\Omega}^{2}$ (b) and $\mathbf{S}^{2}$ (c). The bottom row shows $\mathrm{R}(\mathrm{d})$, and its constituents: $-\operatorname{det}(\mathbf{S})(\mathrm{e})$ and $\operatorname{tr}\left(\boldsymbol{\Omega}^{2} \mathbf{S}\right)(\mathrm{f})$. Results are displayed on a logarithmic scale, with a different color bar adopted for the two positive quantities in (b) and (c).

a popular resource for studying flow topologies [36, 37]. We begin by looking at two basic quantities to derive from $\mathbf{A}$ given both the restricted Euler analysis of the velocity gradient tensor [20], and flow visualization studies [17]: $\mathrm{Q}$ and $\mathrm{R}$ as defined in (7). The fields for these scalars on a sample plane, as well as their constitutive terms as defined in (7) are given in Fig. 3. For ease of display, we employ a modified logarithmic function for the scalar quantities that have both positive and negative values:

$$
\log ^{*}(x)=\left\{\begin{array}{cc}
0 & \text { if } \log _{10}(|x|) \leq 0 \\
\operatorname{sgn}(x) \times \log _{10}(|x|) & \text { if } \log _{10}(|x|)>0
\end{array}\right.
$$




\section{A. The structure of the $Q-R$ diagram}

In Fig. 4 we show the probability distribution functions for $\mathrm{Q}$ and $\mathrm{R}$ for both the original data (solid lines) and the median for thirty nine surrogates (dotted lines) generated for each of the sampled points in the original fields. The small bump at the origin is an artefact of the truncation of values that are negative in their logarithm as seen in (25). The level of agreement between data and surrogates is very good, with a slight tendency for the typical positive value of $\mathrm{Q}$ for the surrogates to be somewhat more positive than for the original data, and for a negative value for $\mathrm{R}$ for the surrogates to be somewhat more negative. We note that this logarithmic scale was used to highlight the small differences that may arise. On a linear scale, no differences are visible to the naked eye. Hence, without conditioning on these terms explicitly, we have a good replication of their overall values.

Given this approximate preservation of the properties of the marginal distributions for $\mathrm{Q}$ and R, we may examine the difference between actual and synthetic HIT for the joint distribution of these terms. That is, are there significant features of actual turbulence in $\mathrm{Q}-\mathrm{R}$ space compared to the synthetic tensors with appropriate values for the marginals? The joint probability distribution functions for the original data and the synthetic tensors, as well as their difference are shown in Fig. 5. While the qualitative shape of the synthetic data distribution function is similar to that for the original data, there is a clear pattern to the contour map of the differences, with the original data exhibiting a clear excess close to the origin and along the Vieillefosse tail [1] at high positive $\mathrm{R}$ and negative $\mathrm{Q}$, as well as a weak excess in the opposite quadrant (negative $\mathrm{R}$ and positive $\mathrm{Q}$ ).

Thus, while previous work has shown that the Vieillefosse tail is a significant property of turbulence relative to synthetic, Gaussian random tensors [38], we can also state that the overall "teardrop" shape is a feature of real HIT compared to locally derived synthetic tensors with appropriate marginal distributions for $\mathrm{Q}$ and $\mathrm{R}$.

The excess concentration at the origin and on the Vieillefosse tail may be explained with respect to the restricted Euler approximation to the dynamics in $\mathrm{Q}-\mathrm{R}$ space [1], and the linear damping closure that extends this model by incorporating a simple model for the

combined action of the viscous and pressure Hessian terms as being proportional to the product of the inverse relaxation timescale, $\tau_{0}$, and the tensor itself [39]. The restricted 


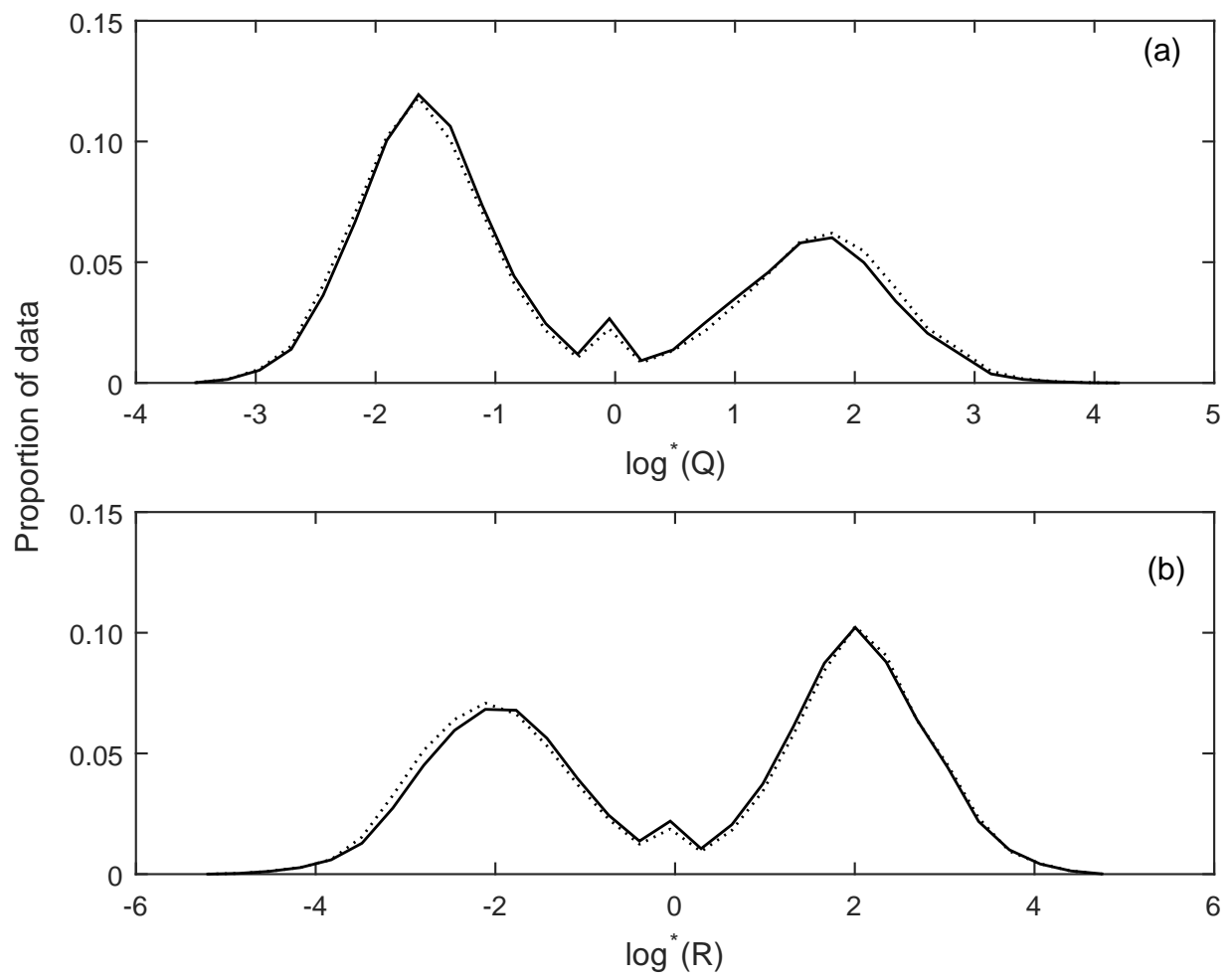

FIG. 4. Histograms of $\log ^{*}(\mathrm{Q})(\mathrm{a})$ and $\log ^{*}(\mathrm{R})(\mathrm{b})$ for the original data (solid lines) and the median values for the surrogate data at each resolved location (dotted lines). The log* function is defined in eq. 25.

Euler system is given by

$$
\frac{d \mathrm{Q}}{d t}=-3 \mathrm{R}, \quad \frac{d \mathrm{R}}{d t}=\frac{2}{3} \mathrm{Q}^{2}
$$

and eliminating $t$, and then solving for $\mathrm{Q}$, one finds that the discriminant function is a time invariant of the dynamics, with convergence from both above and below (see Fig. 3a of the Meneveau review [12]). The linear dampling system in $\mathrm{Q}-\mathrm{R}$ space is

$$
\frac{d \mathrm{Q}}{d t}=-3 \mathrm{R}-2 \mathrm{Q} / \tau_{0}, \quad \frac{d \mathrm{R}}{d t}=\frac{2}{3} \mathrm{Q}^{2}-3 \mathrm{R} / \tau_{0} .
$$

In this case, the origin becomes a stable node, and a saddle point develops at $M$ with coordinates $\left(\mathrm{Q}=-3 \tau_{0}^{-2}, \mathrm{R}=2 \tau_{0}^{-3}\right.$ ), on the Vieillefosse tail. Figure $3 \mathrm{~b}$ in the Meneveau paper shows trajectories of this system of equations and also that for $\mathrm{R}>M$ and when approaching the Vieillefosse tail from above, the trajectories are approximately orthogonal 
(a)
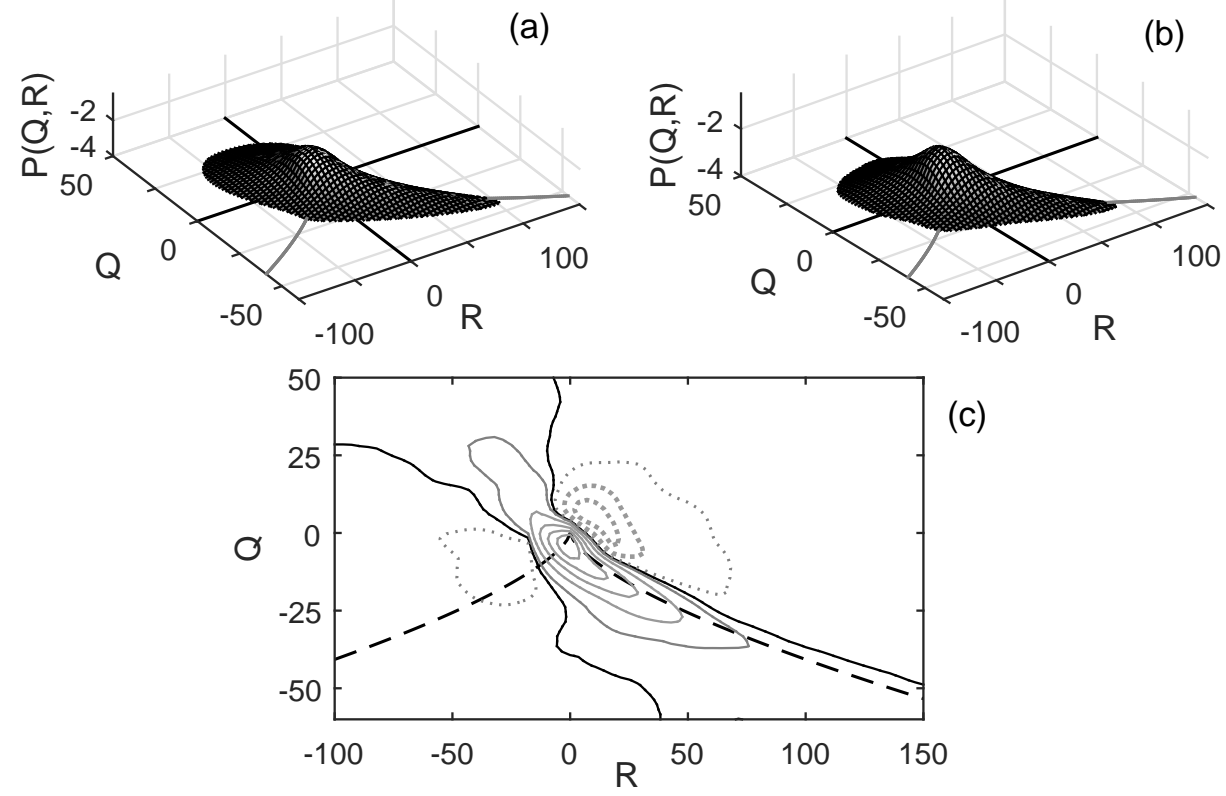

(b)

(c)

FIG. 5. Joint distribution functions for Q and R for the JHU database (a) and its synthetic tensors (b). Contours of the difference are given in (c). In this panel, the zero line contour is given by a black line with positive contours (excess for the original data) as solid lines and negative as dotted. Contours are at $\pm 2.5 \times 10^{-4}, \pm 1 \times 10^{-3}$ and then in intervals of $\pm 1 \times 10^{-3}$. The values are non-dimensionalized using the square $(\mathrm{Q})$ and cube $(\mathrm{R})$ of the Kolmogorov time. The discriminant function separating real and conjugate pair eigenvalues, $\mathrm{Q}^{3}+(27 / 4) \mathrm{R}^{2}=0$, is shown as a gray or dashed line in the upper panels and panel (c), respectively.

to the Vieillefosse tail, a much steeper angle than occurs for the trajectories that approach from below. For $0<\mathrm{R}<M$ trajectories from above are attracted to the fixed point at the origin, while for $\mathrm{R}>M$ they approach the discriminant and are then subject to the finite time singularity. Hence, one would anticipate that the actual data exhibit an excess along the Vieillefosse tail, with a sharper interface between regions of excess and deficit above rather than below the Vieillefosse tail, which is precisely what is observed in Fig. 5.

The topological interpretation of the $\mathrm{Q}$ - $\mathrm{R}$ diagram indicates that the top-left quadrant is a stable focus region where the flow is extensional [24]. There is large positive enstrophy production in this region as a consequence [38]. It has been known since the work of Taylor [40] that $\left\langle\operatorname{tr}\left(\boldsymbol{\Omega}^{2} \mathbf{S}\right)\right\rangle>0$ in turbulence, and the relative excess for the original data in this 
region is therefore to be expected. In contrast, the surrogate data, preferentially occur in the bottom-left and top-right quadrants, which are the stable node with saddles, and unsteady focus with compression regions, respectively [24].

\section{B. Significant differences regarding the eigenvalues of the strain tensor}

A key result from the early direct numerical simulations of homogeneous, isotropic turbulence was that the second eigenvalue of the strain rate tensor is typically positive, as mentioned above [35]. To examine this aspect of turbulence structure, we adopt the Lund and Rogers normalization of the second eigenvalue of the strain rate tensor, $e_{L R}$, given by [41]:

$$
e_{L R}=\frac{3 \sqrt{6} \mathrm{R}_{\mathrm{S}}}{\left(-2 \mathrm{Q}_{\mathrm{S}}\right)^{\frac{3}{2}}},
$$

where $Q_{S}$ and $R_{S}$ are the second and third invariants of the characteristic equation for the strain tensor, S. For Gaussian, random velocity gradient tensors, the distribution function for $e_{L R}$ is uniform over the limits $-1 \leq e_{L R} \leq+1$, with no prefered structure. However, for turbulence, there is a strong peak close to +1 , as can be seen in Fig. 6, which means that the prefered state is one where the two positive eigenvalues are equal, and half the magnitude of the negative strain rate eigenvalue. Thus, deformation favours axisymmetric expansion and the development of disc-like structures.

The distribution function for the median of the surrogate data is not flat (dotted line in Fig. 6), as is the case for Gaussian surrogates, but is less sharply peaked than for the actual data. As a consequence, significant differences between the data and surrogates at the $5 \%$ signifcance level (when $e_{L R}$ for the data is greater or less than the values for all the 39 surrogates generated at a particular location) arise preferentially at $e_{L R}=+1$, with a secondary maximum at $e_{L R}=-1$. In Fig. 7 we show histograms for Q, the enstrophy and total strain for all the data, as well as cases where the results are conditioned on the presence of a significant difference, and if $e_{L R}>\frac{1}{3}$ (line with circles) or $e_{L R}<-\frac{1}{3}$ (dotted line). In both these latter cases, the emergence of a significant difference for $e_{L R}$ is associated with a higher proportion of positive values for $\mathrm{Q}$ compared to the full set of points (which are preferentially negative). However, while for the $e_{L R}>\frac{1}{3}$ situation, the positive and negative modes are of very similar probability, for the $e_{L R}<-\frac{1}{3}$ cases there is a strong bias, with the positive mode some three times larger than the negative mode. Separation into the 


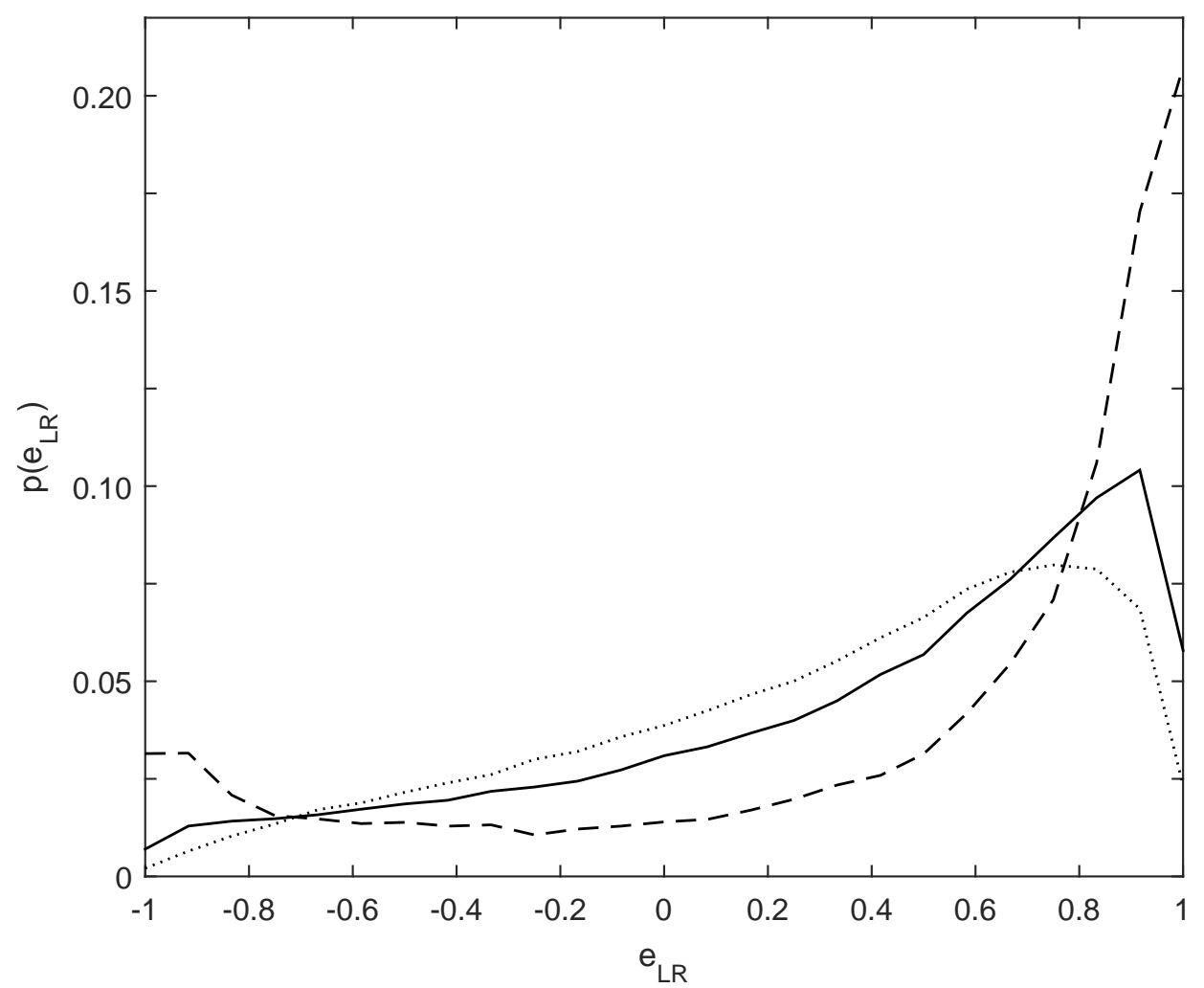

FIG. 6. Histograms for the Lund and Rogers normalization of the strain eigenvalues, $e_{L R}$. The original data are shown as a solid line. The median of the synthetic data is shown as a dotted line. The cases where there is a statistically significant difference between data and surrogates are shown as a dashed line.

component terms (enstrophy and total strain) explains how this tendency results: In Fig. $7 \mathrm{~b}$ it is seen that, irrespective of the tendency towards disc-like $\left(e_{L R}>\frac{1}{3}\right)$ or pencil-like $\left(e_{L R}<-\frac{1}{3}\right)$ structures, there is an excess of enstrophy relative to the unconditioned data. However, the mode for the total strain for the $e_{L R}<-\frac{1}{3}$ cases in Fig. $7 \mathrm{c}$ is less than for the unconditioned data and half an order of magnitude less than for the $e_{L R}>\frac{1}{3}$ cases, resulting in a more clearly defined enstrophy excess and, therefore, positive Q in Fig. 7a.

Related results to those in Fig. 7 are shown in Fig. 8 for $\mathrm{R}$ and its constituent terms: strain production and enstrophy production. The opposite nature of the results for $-s_{i j} s_{j k} s_{k i} \equiv-\operatorname{Det}(\mathbf{S})$ in Fig. 8c follows directly from the conditioning on $e_{L R}$. With negative strain production for $e_{L R}<-\frac{1}{3}$ by definition, given $\mathrm{R}$ is the excess of negative 

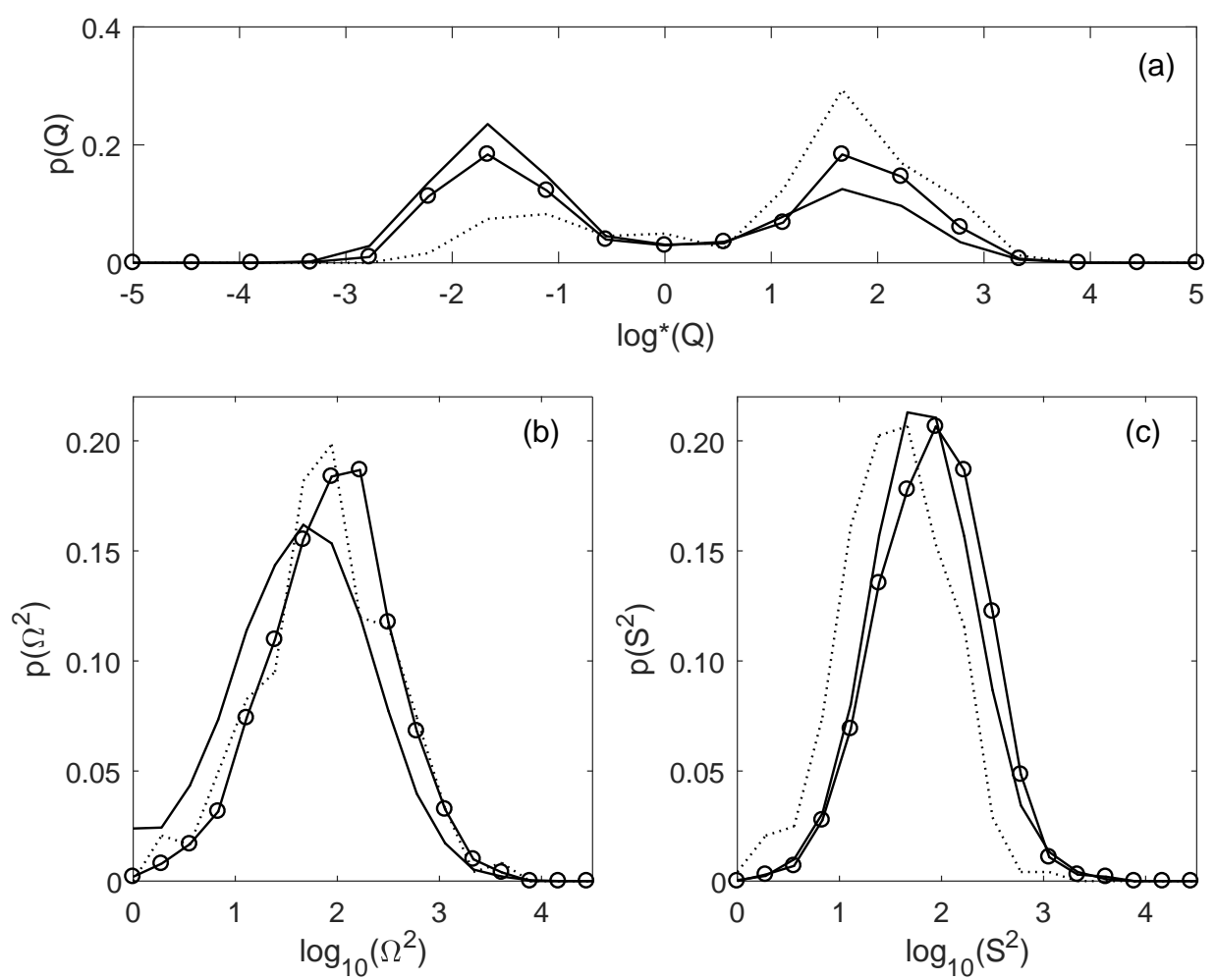

FIG. 7. Histograms for Q (a), enstrophy, $\Omega^{2}(\mathrm{~b})$, and total strain, $S^{2}$ (c) are shown as solid black lines. These quantities conditioned on statistically significant differences for $e_{L R}$ between data and surrogates for $e_{L R}>\frac{1}{3}$ are shown as a black line with circles. Results for statistically significant differences for $e_{L R}$ between data and surrogates for $e_{L R}<-\frac{1}{3}$ are shown as a dotted black line. The $\log ^{*}$ function is defined in eq. 25 .

strain production to enstrophy production (7), we might expect a larger difference in $\mathrm{R}$ than is actually the case. However, we see in Fig. 8b that there is also a preference for negative enstrophy production in these cases, which counteracts the negative strain production to some degree. Thus, it is clear that for the tensors that exhibit statistically significant differences, Taylor's result [40] that $\left\langle\operatorname{tr}\left(\Omega^{2} \mathbf{S}\right)\right\rangle>0$ is clearly coupled to two positive strain eigenvalues and the development of disc-like structures.

The net effect of the differences seen in Fig. 7 and Fig. 8 is to bias the proportional occupancy of the $\mathrm{Q}-\mathrm{R}$ diagram for significant differences in $e_{L R}$ towards $\mathrm{Q}>0, \mathrm{R}<0$ quadrant, in particular, as shown in Table I. Thus, while the differences in the joint PDF emphasize the importance of the Vieillefosse tail (Fig. 5), the opposite quadrant with an 

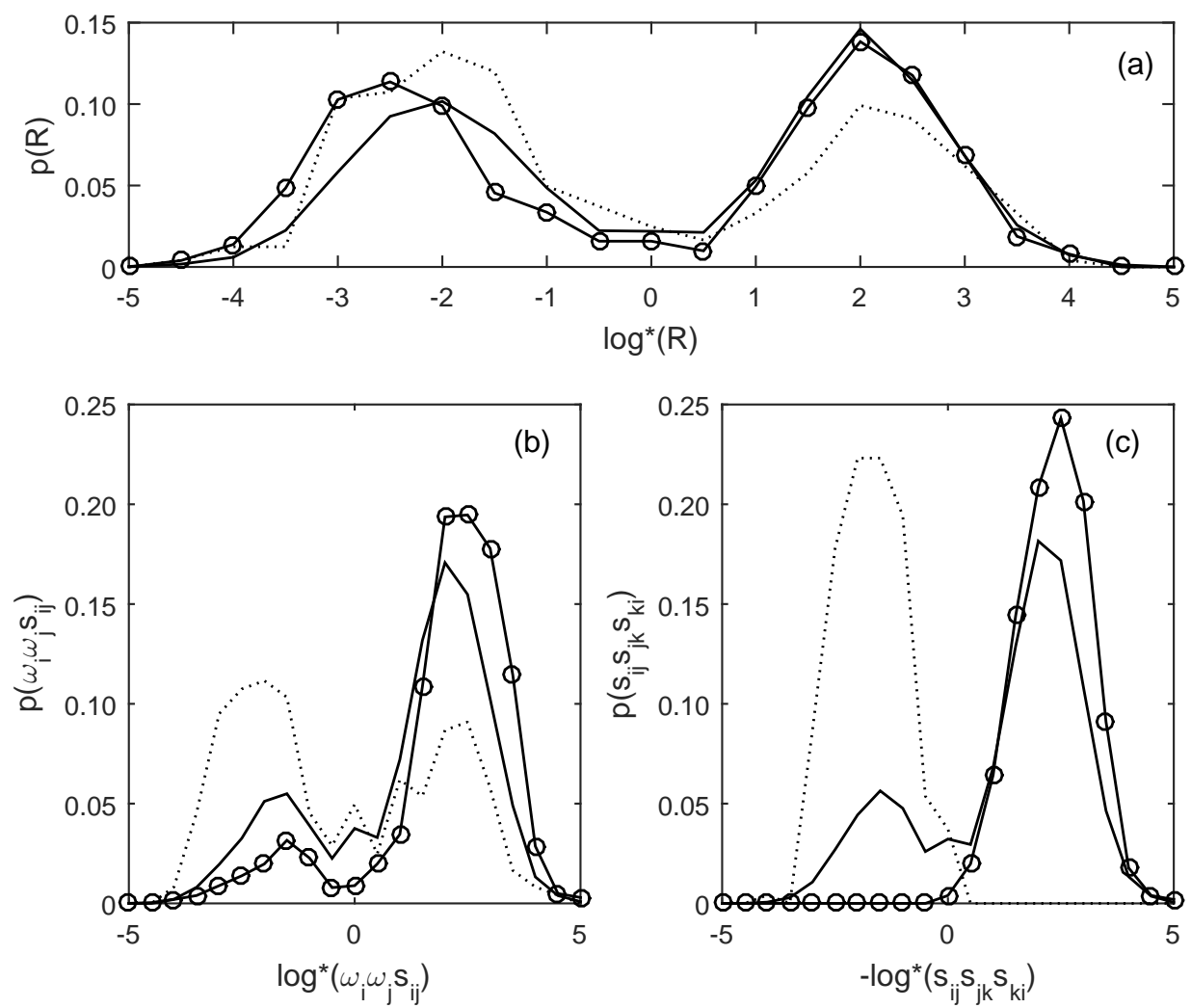

FIG. 8. Histograms for R (a), enstrophy production, $\omega_{i} \omega_{j} s_{i j} \equiv \operatorname{tr}(\boldsymbol{\Omega} \boldsymbol{\Omega} \mathbf{S})$ (b), and strain production, $-s_{i j} s_{j k} s_{k i} \equiv \operatorname{Det}(\mathbf{S})(\mathrm{c})$ are shown as solid black lines. These quantities conditioned on statistically significant differences for $e_{L R}$ between data and surrogates for $e_{L R}>\frac{1}{3}$ are shown as a black line with circles. Results for statistically significant differences for $e_{L R}$ between data and surrogates for $e_{L R}<-\frac{1}{3}$ are shown as a dotted black line. The $\log ^{*}$ function is defined in eq. 25 .

excess occupancy with respect to the synthetic data is where we see the largest effect when we examine significantly different strain behavior.

This result is decomposed further in Table II where the relative occupancy of three equal interval classes for $e_{L R}$ are analyzed as a function of the region of the $\mathrm{Q}-\mathrm{R}$ diagram for the full dataset and for the significant cases. In the middle range of values for $e_{L R}$ in the third column, the second eigenvalue is close to zero and it is rare to generate significant differences (Fig. 6) in this region. Hence, we see a decrease in proportional occupancy throughout this column, which is largely reallocated to positive $e_{L R}$ (axisymmetric expansion).

We see that the only region of $\mathrm{Q}-\mathrm{R}$ space with an approximate equipartition of the 
TABLE I. Percentage occupancy of different parts of the $\mathrm{Q}-\mathrm{R}$ diagram

\begin{tabular}{lcc}
\hline & Full dataset Significant $e_{L R}$ \\
\hline $\mathrm{R}>0, \mathrm{Q}>0$ & 11.0 & 14.9 \\
$\mathrm{R}<0, \mathrm{Q}>0$ & 26.0 & 39.3 \\
$\mathrm{R}<0, \mathrm{Q}<0, \Delta>0$ & 9.7 & 9.0 \\
$\mathrm{R}<0, \mathrm{Q}<0, \Delta<0$ & 9.0 & 4.2 \\
$\mathrm{R}>0, \mathrm{Q}<0, \Delta>0$ & 12.6 & 16.2 \\
$\mathrm{R}>0, \mathrm{Q}<0, \Delta<0$ & 30.2 & 16.4 \\
\hline
\end{tabular}

TABLE II. Relative frequency (expressed as a percentage) of different $e_{L R}$ states for various parts of the $\mathrm{Q}-\mathrm{R}$ diagram for the full dataset and the significant cases (shown in brackets). The percentages are calculated over the columnwise statistics such that each row sums to $100 \%$.

\begin{tabular}{lccc}
\hline & $-1 \leq e_{L R}<-\frac{1}{3}-\frac{1}{3} \leq e_{L R}<\frac{1}{3}$ & $\frac{1}{3} \leq e_{L R} \leq 1$ \\
\hline $\mathrm{R}>0, \mathrm{Q}>0$ & $29.2(35.6)$ & $35.7(19.8)$ & $35.1(44.6)$ \\
$\mathrm{R}<0, \mathrm{Q}>0$ & $14.0(15.5)$ & $27.4(13.1)$ & $58.6(71.4)$ \\
$\mathrm{R}<0, \mathrm{Q}<0, \Delta>0$ & $31.1(34.6)$ & $30.8(16.7)$ & $38.1(48.7)$ \\
$\mathrm{R}<0, \mathrm{Q}<0, \Delta<0$ & $26.8(20.9)$ & $48.0(31.2)$ & $25.2(47.9)$ \\
$\mathrm{R}>0, \mathrm{Q}<0, \Delta>0$ & $3.6(3.7)$ & $13.5(5.9)$ & $82.9(90.4)$ \\
$\mathrm{R}>0, \mathrm{Q}<0, \Delta<0$ & $0.8(1.6)$ & $15.7(6.6)$ & $83.5(91.8)$ \\
\hline
\end{tabular}

decrease in the $-\frac{1}{3} \leq e_{L R}<\frac{1}{3}$ regime to the negative and positive cases $(6.4: 9 . .5)$ is that in the top row of Table II where $\mathrm{R}>0, \mathrm{Q}>0$ and the flow is in an unsteady focus with compression regime in the terminology of Ooi et al. [24]. Thus, the preferential tendency for the significant results to form tubes more often than discs relative to any other region of Q-R space is consistent with this topology. The only region that exhibits a relative decrease in the $-1 \leq e_{L R}<-\frac{1}{3}$ regime between the original data and the significant points is the opposite region in the fourth row $(\mathrm{R}<0, \mathrm{Q}<0, \Delta<0)$, which is a stable node-saddle-saddle. With this particularly strong tendency for two positive strain eigenvalues for the significant events compared to the other $\mathrm{R}<0$ regions resulting in stronger strain production, the negative value for $\mathrm{R}$ is a particular consequence of strong enstrophy production in this region for the significant cases. 


\section{CONCLUSION}

High quality, eddy-resolving numerical simulations, and three-dimensional, tomographic PIV make the study of scalars derived from the velocity gradient tensor more accessible to analysis than ever before. Because for inhomogeneous or anisotropic flows, such as those found in many practical situations, we do not have the depth of understanding to know what the expected behavior of, for example, enstrophy production versus strain production, or vorticity vector alignment with strain eigenvectors will be, testing the statistical significance of observed behaviors with respect to suitable null models provides an alternate means to progress. Gaussian random matrices have been used in the past for this purpose when studying HIT, but do not provide a particularly physically relevant null hypothesis and may only be applied in a bulk fashion as they are not constrained to the actual behavior of the individual, observed tensor. This limits the relevance of this approach to inhomogeneous phenomena. In this study we have presented a point-specific methodology that is constrained to mathematical bounds on the non-normality of the observed tensor. By placing the direct constraint on a mathematical rather than a physical property, we can test hypotheses regarding the behavior of the physical quantities.

While perhaps our approach to turbulence analysis is most useful for complex flows, HIT provides a case where we can both (a) test that our technique works and (b) refine previous understanding of what aspects of the velocity gradient tensor are true features of the turbulence by considering these properties with respect to our more carefully constrained, synthetic, surrogate tensors. Undertaking two analyses using our technique applied to HIT has provided several results:

- The teardrop shape of the joint distribution function in $\mathrm{Q}-\mathrm{R}$ space is largely due to an excess probability both along the Vieillefosse tail and in the upper left $(\mathrm{Q}>0, \mathrm{R}<0)$ quadrant; and,

- Significantly different values for the Lund and Rogers [41] normalization of the strain rate eigenvalues also occur preferentially in this $\mathrm{Q}>0, \mathrm{R}<0$ quadrant as seen in Table I. Here, as well as close to the Vieillefosse tail and, in particular, in the $(\mathrm{Q}<0, \mathrm{R}<$ $0, \Delta<0)$ region, there is a clear tendency for these significant events to preferentially favour an axisymmetric expansive state, favoring the formation of disc-like structures 
in the flow field;

- While all regions of the $\mathrm{Q}-\mathrm{R}$ diagram have the significant cases preferentially exhibiting a tendency to axisymmetric expansion, this is least strong in the $\mathrm{Q}>0, \mathrm{R}>0$ region, where vortex compression leads to dissipative events [38, 42].

Hence, our technique provides a means to ask questions of such data and explore significance relative to random tensors that are not purely kinematic but also capture aspects of the relevant dynamics. With ever more sophisticated models of the dynamics of the velocity gradient tensor being developed [43,44], and topological analyses being used to elucidate the physics of more complex flows [45, 46], we anticipate that a hypothesis testing framework as outlined here, will be of increasing utility.

\section{ACKNOWLEDGMENTS}

This research was supported by a Royal Academy of Engineering/Leverhulme Trust Senior Research Fellowship LTSRF1516-12-89 awarded to the author. He is grateful to the hospitality of the Department of Aeronautics, Imperial College London and the Department of Mechanical Engineering at Johns Hopkins University where this work was largely undertaken.

[1] P. Vieillefosse, "Internal motion of a small element of fluid in an inviscid flow," Physica A 125, 150-162 (1984).

[2] L. Shtilman, M. Spector, and A. Tsinober, "On some kinematic versus dynamic properties of homogeneous turbulence," J. Fluid Mech. 247, 65-77 (1993).

[3] A. Tsinober, L. Shtilman, and H. Vaisburd, "A study of properties of vortex stretching and enstrophy generation in numerical and laboratory turbulence," Fluid Dyn. Res. 21, 477-494 (1997).

[4] J. Theiler, S. Eubank, A. Longtin, B. Galdrikian, and J.D. Farmer, "Testing for nonlinearity in time series: the method of surrogate data," Physica D 58, 77-94 (1992).

[5] T. Schreiber and A. Schmitz, "Improved surrogate data for nonlinearity tests," Phys. Rev. Lett. 77, 635-638 (1996). 
[6] T. Schreiber and A. Schmitz, "Discrimination power of measures for nonlinearity in a time series," Phys. Rev. E 55, 5443-5447 (1997).

[7] C. J. Keylock, "A wavelet-based method for surrogate data generation," Physica D 225, 219228 (2007).

[8] C. J. Keylock, "Characterizing the structure of nonlinear systems using gradual wavelet reconstruction," Nonlinear Proc.Geophys. 17, 615-632 (2010).

[9] C. J. Keylock, R. Stresing, and J. Peinke, "Gradual wavelet reconstruction of the velocity increments for turbulent wakes," Phys. Fluids 27, 025104 (2015).

[10] C. J. Keylock, T. E. Tokyay, and G. Constantinescu, "A method for characterising the sensitivity of turbulent flow fields to the structure of inlet turbulence," J. Turbul. 12, N45 (2011).

[11] C. J. Keylock, "Multifractal surrogate-data generation algorithm that preserves pointwise Holder regularity structure, with initial applications to turbulence," Phys. Rev. E 95, 032123 (2017).

[12] C. Meneveau, "Lagrangian dynamics and models of the velocity gradient tensor in turbulent flows," Ann. Rev. Fluid Mech. 43, 219-245 (2011).

[13] B. Ganapathisubramani, E. K. Longmire, I. Marusic, and S. Pothos, "Dual-plane PIV technique to determine the complete velocity gradient tensor in a turbulent boundary layer," Exp. Fluids 39, 222-231 (2005).

[14] O. R. H. Buxton, S. Laizet, and B. Ganapathisubramani, "The effects of resolution and noise on kinematic features of fine-scale turbulence," Exp. Fluids 51, 1417-1437 (2011).

[15] R. M. Kerr, "Higher-order derivative correlations and the alignment of small-scale structures in isotropic, numerical turbulence," J. Fluid Mech. 153, 31-58 (1985).

[16] J. C. R. Hunt, A. A. Wray, and P. Moin, Eddies, stream, and convergence zones in turbulent flows, Tech. Rep. CTR-S88 (Center for Turbulence Research, Stanford University, 1988).

[17] Y. Dubief and F. Delcayre, "On coherent-vortex identification in turbulence," J. Turbul. 1 (2000).

[18] W. Y. Chang, G. Constantinescu, W. F. Tsai, and H. C. Lien, "Coherent structure dynamics and sediment erosion mechanisms around an in-stream rectangular cylinder at low and moderate angles of attack," Water Resour. Res. 47 (2011), 10.1029/2011WR010586.

[19] C. M. De Silva, J. Philip, and I. Marusic, "Minimization of divergence error in volumetric 
velocity measurements and implications for turbulence statistics," Exp. Fluids 54, 1-17 (2013).

[20] B. J. Cantwell, "Exact solution of a restricted Euler equation for the velocity gradient tensor," Phys. Fluids A 4, 782-793 (1992).

[21] K. K. Nomura and G. K. Post, "The structure and dynamics of vorticity and rate of strain in incompressible homogeneous turbulence," J. Fluid Mech. 377, 65-97 (1998).

[22] H. Liu and E. Tadmor, "Spectral dynamics of the velocity gradient field in restricted flows," Commun. Math. Phys. 228, 435-466 (2002).

[23] L. Biferale, L. Chevillard, C. Meneveau, and F. Toschi, "Multiscale model of gradient evolution in turbulent flows," Phys. Rev. Lett. 98, 214501 (2007).

[24] A. Ooi, J. Martin, J. Soria, and M. S. Chong, "A study of the evolution and characteristics of the invariants of the velocity-gradient tensor in isotropic turbulence," J. Fluid Mech. 381, 141-174 (1999).

[25] Y. Zhou, K. Nagata, Y. Sakai, H. Suzuki, Y. Ito, O. Terashima, and T. Hayase, "Development of turbulence behind the single square grid," Phys. Fluids 26, 045102 (2014).

[26] S. Laizet, J. Nedić, and C. Vassilicos, "Influence of the spatial resolution on fine-scale features in DNS of turbulence generated by a single square grid," Int. J. Comp. Fluid Dyn. 29, 286-302 (2015).

[27] C. Escauriaza and F. Sotiropoulos, "Lagrangian model of bed-load transport in turbulent junction flows," J. Fluid Mech. 666, 36-76 (2011).

[28] C. J. Keylock, K. S. Chang, and G. S. Constantinescu, "Large eddy simulation of the velocityintermittency structure for flow over a field of symmetric dunes," J. Fluid Mech. 805, 656-685 (2016).

[29] Y. Li, E. Perlman, M. Wan, Y. Yang, R. Burns, C.Meneveau, S. Chen, A. Szalay, and G. Eyink, "A public turbulence database cluster and applications to study lagrangian evolution of velocity increments in turbulence," J. Turbulence 9 (2008).

[30] F. Scarano and C. Poelma, "Three-dimensional vorticity patterns of cylinder wakes," Exp. Fluids 47, 69-83 (2009).

[31] I. Schur, "On the characteristic roots of a linear substitution with an application to the theory of integral equations (in German)," Math. Ann. 66, 488-510 (1909).

[32] P. Henrici, "Bounds for iterates, inverses, spectral variation and fields of values of non-normal matrices," Numer. Math. 4, 24-40 (1962). 
[33] P. J. Eberlein, "On measures of non-normality for matrices," Amer. Math. Mon. 72, 995-996 (1965).

[34] G. H. Golub and C. F. van Loan, Matrix Computations, 4th ed. (Johns Hopkins University Press, 2013).

[35] W. T. Ashurst, A. R. Kerstein, R. A. Kerr, and C. H. Gibson, "Alignment of vorticity and scalar gradient with strain rate in simulated Navier-Stokes turbulence," Phys. Fluids 30, 2343-2353 (1987).

[36] M. Wan, Z. Xiao, C. Meneveau, G. L. Eyink, and S. Chen, "Dissipation-energy flux correlations as evidence for the Lagrangian energy cascade in turbulence," Phys. Fluids 22, 1-4 (2010).

[37] J. M. Lawson and J. R. Dawson, "On velocity gradient dynamics and turbulent structure," J. Fluid Mech. 780, 60-98 (2015).

[38] A. Tsinober, "Vortex stretching versus production of strain/dissipation," in Turbulence Structure and Vortex Dynamics, edited by J. C. R. Hunt and J. C. Vassilicos (Cambridge University Press, 2001) pp. 164-191.

[39] J. Martin, C. Dopazo, and L. Valiño, "Dynamics of velocity gradient invariants in turbulence: restricted Euler and linear diffusion models," Phys. Fluids 10, 2012-25 (1998).

[40] G. I. Taylor, "The spectrum of turbulence," Proc. R. Soc. Lond. A 164, 476-490 (1938).

[41] T. S. Lund and M. M. Rogers, "An improved measure of strain state probability in turbulent flows," Phys. Fluids 6, 1838-1847 (1994).

[42] R. Betchov, "An inequality concerning the production of vorticity in isotropic turbulence," J. Fluid Mech. 1, 497-504 (1956).

[43] M. Wilczek and C. Meneveau, "Pressure Hessian and viscous contributions to velocity gradient statistics based on Gaussian random fields," J. Fluid Mech. 756, 191-225 (2014).

[44] P. L. Johnson and C. Meneveau, "A closure for Lagrangian velocity gradient evolution in turbulence using recent-deformation mapping of initially Gaussian fields," J. Fluid Mech. 804, 387-419 (2016).

[45] R. Gomes-Fernandes, B. Ganapathisubramani, and J. C. Vassilicos, "Particle image velocimetry study of fractal-generated turbulence," J. Fluid Mech. 711, 306-336 (2012).

[46] Y. Zhou, K. Nagata, Y. Sakai, and Y. Ito, "Spatial evolution of the helical behavior and the 2/3 power-law in single-square-grid-generated turbulence," Fluid Dyn. Res. 48, 021404 
(2016). 